\title{
Alimentación de cerdos en levante y ceba utilizando bore (Alocasia macrorhyza) y aceite de palma
}

Feed swine in growing and finishing used Alocasia macrorhyza and palm oil

\author{
Van Doren Marcos ${ }^{1}$, Giraldo Rodrigo ${ }^{1}$, Álvarez Ernesto ${ }^{1} \mathrm{y}$ \\ Hurtado Nery Víctor Libartdo \\ ${ }^{1}$ Médicos Veterinarios Zootecnistas, ${ }^{2}$ Profesor Universidad de los Llanos, km 12 \\ Vía Apiay, Villavicencio, Colombia \\ vhurtado@unillanos.edu.co
}

Recibido 03 Julio 2012, Aceptado10 Octubre2012

\section{RESUMEN}

Este trabajo fue realizado con el objetivo de evaluar el efecto de la inclusión de Bore (Alocasia macrorhyza) en la ración de cerdos en las fases de levante y ceba sobre el consumo de alimento, ganancia diaria de peso y conversión alimenticia. El estudio se realizó en el Municipio de Granada (Meta), con 24 cerdos de raza Pietrain, distribuidos en 4 tratamientos de dos cerdos cada uno, con tres repeticiones. Los tratamientos fueron: Tratamiento testigo, el cual corresponde a un núcleo comercial con aceite de palma, CT; bore a voluntad + núcleo proteico de $46.9 \%$ proteína bruta, NPB; bore a voluntad + núcleo proteico $+0.1 \mathrm{~kg}$ de aceite de palma, NPB-AP100; bore, a voluntad + núcleo proteico $+0.2 \mathrm{~kg}$ de aceite de palma NPB-AP200. Los niveles de bore influyeron significativamente $(P<0.05)$ sobre la ganancia diaria de peso. En la fase de crecimiento la ganancia diaria de peso fue de $0.52,0.66,0,68$ y $0.68 \mathrm{~kg}$ y en la fase de ceba de $0.77,0.75,0.76$ y $0.76 \mathrm{~kg}$ respectivamente. En conclusión, el suministro de bore a voluntad y núcleo proteico con inclusión hasta de $0.2 \mathrm{~kg}$ de aceite de palma mejora la ganancia diaria de peso de cerdos en crecimiento y ceba.

Palabras clave: Alimentos alternativos, bore, ganancia de peso, cerdos.

\begin{abstract}
This research was carried with the purpose of to evaluate the effects of bore (Alocasia macrorhyza) in the diet of grower and finishing pigs on feed intake, gain
\end{abstract}


of weight and ratio intake/gain of weight. The study was carried in Granada, with 24 pigs Pietrain distributed in 4 treatments of two pigs each one, with three repetitions. The treatments were: Control treatment, CT, which corresponds to a commercial nucleus with palm oil; Alocasia macrorhyza to will + nucleus protein of 46.9 crude protein, NPB; Alocasia macrorhyza to will + nucleus protein $+0.1 \mathrm{~kg}$ of oil of palm, NPB-AP100 and Alocasia macrorhyza to will + nucleus protein $+0.2 \mathrm{~kg}$ oil of palm, NPB-AP200. The levels of Alocasia macrorhyza had effects statistic $(P<0.05)$ on gain of weight. The gains of weight were $0.52,0.66,0.68$ y $0.68 \mathrm{~kg}$ in swine growing phase and de $0.77,0.75,0.76$ y $0.76 \mathrm{~kg}$ in swine finishing phase respectively. In conclusion, the supply ad libitum of Alocasia macrorhyza and nucleus protein with oil of palm to $0.2 \mathrm{~kg}$ improvement the gain of weight of the swine in growing and finishing phases.

Keywords: Food alternatives, bore, gain of weight, swine.

\section{INTRODUCCIÓN}

La industria porcina se ha convertido en una de las formas más eficientes de producir proteína animal para consumo humano. En forma convencional los cerdos han sido alimentados con productos concentrados, preparados con materias primas que compiten con la alimentación humana, principalmente maíz, soya y arroz, y que además incrementan los costos de producción.

En zonas de economía campesina se han utilizado diferentes ingredientes alternativos en la alimentación de los cerdos como yuca y plátano (Garzón, 2003), jugo de caña y harina de follaje de morera para cerdos en crecimiento (González et al., 2006), suero de leche (Hauptli et al., 2005) para lechones en precebos, azolla y jugo de caña para cerdos en crecimiento (Pinto-Santini et al., 2005), follaje fresco de morera para cerdos en ceba (Esquijerosa et al., 2008), y aceite crudo de palma para cerdos en fase de engorde (Bermúdez y Rodríguez, 1998).

La composición nutricional del Bore (Alocasia macrorhyza) depende de la parte de la planta que se suministra a los animales, como la raíz, el tallo, la hoja completa, el pecíolo o parénquima foliar. El tallo del bore contiene $16.3 \%$ de materia seca y 
$6.9 \%$ de proteína bruta (Bastos, 1995). Estos valores permiten la utilización potencial del bore en sistemas sostenibles de producción de cerdos a pequeña escala.

Garzón, (1997) propone la alimentación de cerdos con recursos obtenidos en la finca o la región y recomienda el suministro a voluntad de hojas, de tallos y raíces picadas de bore a cerdos en levante y ceba adicionados de $0.9-1,2$ y $1.2-1.8 \mathrm{~kg}$ respectivamente de concentrado, con el propósito de reducir hasta en $40 \%$ los costos de producción sin afectar el rendimiento del cerdo. Rodríguez et al., (2006), trabajando cerdos de 25 a $56 \mathrm{~kg}$, alimentados con $4 \mathrm{~kg}$ diarios de bore y 250 gramos de torta de soya, no encontraron diferencia significativa en ganancia de peso y conversión alimenticia, con animales alimentados con 500 gramos diarios de torta de soya y jugo de caña de azúcar.

Los resultados de desempeño de los cerdos alimentados con bore dependen de la forma de uso de la planta, después de tratar la raíz de taro (Alocasia macrorrhiza), Aráceae de la misma familia del bore, con hidróxido de sodio constataron que se puede incorporar hasta en $50 \%$ en dietas para cerdos en crecimiento. El bore también ha sido utilizado en forma de harina en la alimentación de conejos en crecimiento con niveles de inclusión hasta de $25 \%$ de la dieta (Pino y Peña, 2009).

Este trabajo tuvo por objetivo evaluar el efecto de la inclusión del tallo de bore cocido y aceite de palma, en la alimentación de cerdos en levante y ceba, sobre el consumo de alimento, ganancia de peso y conversión alimenticia.

\section{MATERIALES Y MÉTODOS}

Localización. El trabajo se realizó en la finca de un pequeño productor de cerdos ubicada en el Municipio de Granada; que se encuentra localizado a 378 m.s.n.m, con temperatura promedio de $29^{\circ} \mathrm{C}$, en piso térmico cálido, con latitud Norte $3^{\circ} 32$ " y Longitud Oeste $73^{\circ} 44^{\prime \prime}$; en el Departamento del Meta, Colombia.

Animales experimentales. Fueron utilizados 24 cerdos Pietrain de $31.2 \pm 1.72 \mathrm{~kg}$ de peso, distribuidos en un diseño experimental de bloques al azar con 4 
tratamientos, tres repeticiones y dos animales por repetición, la unidad experimental estaba constituida por un cerdo. Los tratamientos fueron: Tratamiento control, TC; Núcleo proteico de 46.9\% PB, + Bore a voluntad, NPB; Núcleo proteico de $46.9 \%$ PB + $0.1 \mathrm{~kg}$ de Aceite de Palma + Bore a voluntad, NPB-AP100 y Núcleo proteico de 46.9\% PB + $0.2 \mathrm{~kg}$ de Aceite de Palma + Bore a voluntad, NPB-AP200.

Manejo de animales. Los cerdos se acostumbraron durante una semana al consumo del bore. Las raciones experimentales se formularon para dos fases, de levante de 30 a $50 \mathrm{k}$. y ceba de $50 \mathrm{~kg}$ hasta el peso del mercado. Los cerdos fueron pesados al inicio y quincenalmente para estimar la obtención de peso de la fase correspondiente. La fase experimental tuvo una duración de 84 días, dividida en dos periodos de 42 días cada uno. El bore fue suministrado a partir de las 9:00 a.m, durante 3 a 4 veces al día dependiendo del consumo, el cual era pesado antes y después del suministro y al final del día se totalizaba el consumo de cada grupo. En las tablas 1 y 2 se presenta la composición centesimal de las dietas experimentales. El suministro de ración y el aceite vegetal utilizados en este ensayo se realizó dos veces al día, en horas de la mañana y por la tarde.

Análisis estadístico. Las variables consumo diario de ración, ganancia de peso y conversión alimenticia en las fases evaluadas fueron sometidos a análisis de varianza y las medias comparadas por la Prueba de Tukey al $5 \%$ de probabilidad.

\section{RESULTADOS Y DISCUSIÓN}

En las Tablas 3, 4 y 5 se muestran los parámetros zootécnicos evaluados en la fase de levante y ceba de cerdos alimentados con bore a voluntad suplementado con núcleo proteico e inclusión de aceite de palma.

El consumo diario de alimento se aumentó significativamente $(P<0.01)$ en el tratamiento con bore a voluntad y núcleo proteico, la adición de aceite de palma no influyó en el consumo diario de alimento de cerdos en levante (Tabla 3). El suministro de bore a voluntad + núcleo proteico con o sin adición de aceite de palma aumentó significativamente $(\mathrm{P}<0.01)$ la ganancia de peso (Tabla 3$)$, de 
cerdos en la fase de levante. Este resultado está relacionado con el consumo diario de ración, en la cual el incremento en la ingestión de nutrientes se transforma en tejido corporal, lo cual se refleja en el aumento de la ganancia de peso diario.

Tabla 1. Composición centesimal de las raciones experimentales para levante

\begin{tabular}{|c|c|c|c|c|}
\hline Componente & TC & NPB & NPB-AP100 & NPB-AP200 \\
\hline Harina de Carne & 13.5 & 21.0 & 20.1 & 21.0 \\
\hline Torta de Soya & 17.8 & 73.4 & 73.40 & 73.4 \\
\hline Harina de Arroz & 12.3 & - & - & - \\
\hline Torta de Palmiste & 30.6 & - & - & - \\
\hline Tricalfos & 1.53 & 3.16 & 3.16 & 3.16 \\
\hline Carbonato de Calcio & 0.76 & 0.47 & 0.47 & 0.47 \\
\hline Lisina & 0.08 & 0.39 & 0.39 & 0.39 \\
\hline Metionina & 0.04 & 0.18 & 0.18 & 0.18 \\
\hline Vitamipak & 0.15 & 0.36 & 0.36 & 0.36 \\
\hline Sal & 0.30 & 1.09 & 1.09 & 1.09 \\
\hline Sal & 0.30 & 1.09 & 1.09 & 1.09 \\
\hline Aceite de Palma & 23.1 & - & - & - \\
\hline \multicolumn{5}{|c|}{ Valores nutricionales analizados } \\
\hline Humedad $^{2}$ & - & 8.69 & 8.69 & 8.69 \\
\hline Materia seca ${ }^{2}$ & - & 91.3 & 91.3 & 91.3 \\
\hline Proteína cruda & 21.6 & 46.9 & 46.9 & 46.9 \\
\hline Fibra Cruda & - & 2.15 & 2.15 & 2.15 \\
\hline $\begin{array}{l}\text { Energía } \\
\text { Mcal/kg }\end{array}$ & 3.79 & 4.26 & 4.26 & 4.26 \\
\hline
\end{tabular}

${ }^{2}$ Análisis realizado en el Laboratorio de Nutrición Animal, Universidad de los Llanos 
Tabla 2. Composición centesimal de las raciones experimentales para ceba

\begin{tabular}{|c|c|c|c|c|}
\hline Componente & TC & NPB & NPB-AP100 & NPB-AP200 \\
\hline Harina de Carne & 12.8 & 25.7 & 25.7 & 25.7 \\
\hline Torta de Soya & 16.9 & 66.6 & 66.6 & 66.6 \\
\hline Harina de Arroz & 11.7 & - & - & -- \\
\hline Torta de Palmiste & 29.1 & - & - & - \\
\hline Tricalfos & 1.46 & 4.29 & 4.29 & 4.29 \\
\hline Carbonato de Calcio & 0.73 & 0.64 & 0.64 & 0.64 \\
\hline Lisina & 0.29 & 0.53 & 0.53 & 0.53 \\
\hline Metionina & 0.14 & 0.24 & 0.24 & 0.24 \\
\hline Vitamipak & 0.07 & 0.50 & 0.50 & 0.50 \\
\hline Sal & 0.04 & 1.48 & 1.48 & 1.48 \\
\hline Aceite de Palma & 26.7 & - & - & - \\
\hline \multicolumn{5}{|c|}{ Valores nutricionales analizados } \\
\hline Humedad $^{2}$ & - & 8.27 & 8.27 & 8.27 \\
\hline Materia seca ${ }^{2}$ & - & 91.7 & 91.7 & 91.7 \\
\hline Proteína Cruda² & - & 46.1 & 46.1 & 46.1 \\
\hline Fibra Cruda² & - & 3.69 & 3.69 & 3.69 \\
\hline $\begin{array}{l}\text { Energía } \\
\text { Mcal/kg }\end{array}$ & - & 4.33 & 4.33 & 4.33 \\
\hline
\end{tabular}

${ }^{1}$ Valores estimados

${ }^{2}$ Análisis realizado en el Laboratorio de Nutrición Animal, Universidad de los Llanos

No hubo efecto significativo sobre la conversión alimenticia del consumo a voluntad de bore de cerdos en levante. Sin embargo, el tratamiento con bore a voluntad + núcleo proteico + aceite de palma, NPB-AP100 y NPB-AP200, presentaron mejor conversión alimenticia en relación con los tratamientos sin adición de aceite de palma. 
Tabla 3. Rendimiento zootécnico de cerdos en levante alimentados con bore a voluntad suplementado con núcleo proteico y aceite vegetal

\begin{tabular}{|c|c|c|c|c|c|c|}
\hline Parámetros & $\mathrm{TC}$ & NPB & $\begin{array}{l}\text { NPB - } \\
\text { AP100 }\end{array}$ & $\begin{array}{l}\text { NPB - } \\
\text { AP200 }\end{array}$ & SEM ${ }^{1}$ & $\mathbf{P}^{2}$ \\
\hline Peso inicial & 30 & 31.1 & 31.1 & 33.5 & & \\
\hline Peso final & 52.0 & 58.8 & 59.7 & 62.1 & & \\
\hline Consumo diario, kg & $1.30^{b}$ & $1.80^{\mathrm{a}}$ & $1.49^{b}$ & $1.40^{b}$ & 0.11 & $<0.01$ \\
\hline Ganancia diaria, kg & $0.52^{b}$ & $0.66^{a}$ & $0,68^{a}$ & $0.68^{a}$ & 0.04 & $<0.01$ \\
\hline Conversión Alimenticia & $2.48^{\mathrm{ab}}$ & $2.79^{b}$ & $2.12^{\mathrm{a}}$ & $2.15^{a}$ & 0.16 & $<0.01$ \\
\hline
\end{tabular}

En la fase de ceba el suministro de bore a voluntad + núcleo proteico con o sin inclusión de aceite de palma aumentó significativamente $(P<0.01)$ el consumo diario de ración de cerdos en fase de ceba (Tabla 4), lo que indica que el bore a voluntad estimuló el consumo de diario de ración. La ganancia diaria de peso en relación al testigo no fue influenciada $(P>0,05)$ por el suministro de bore a voluntad + núcleo proteico con inclusión de aceite de palma a cerdos en fase de ceba. El tratamiento control expreso la mejor conversión alimenticia $(P<0,01)$ en la fase de ceba, entre los tratamientos con bore a voluntad con o sin adición de aceite de palma no hubo diferencias significativas en la conversión alimenticia, en los cuales se requiere mayor cantidad de alimento por cada kilogramo de ganancia de peso. Estos resultados explican debido al alto consumo de ración de los tratamientos con adición de bore a voluntad.

En el análisis consolidado de las fases de levante y ceba (Tabla 5) la ganancia de peso diario se incrementó significativamente $(P<0.01)$ en los tratamientos con inclusión de bore a voluntad + núcleo proteico con o sin adición de aceite de palma. En levante y ceba el consumo diario de ración fue mayor en los tratamientos con suministro de bore a voluntad y núcleo proteico, NPB, entre el 
tratamiento testigo y los tratamientos con bore a voluntad con o sin adición de aceite de palma no hubo diferencia significativa.

Tabla 4. Rendimiento zootécnico de cerdos en ceba alimentados con bore a voluntad suplementado con núcleo proteico y aceite vegetal

\begin{tabular}{lcccccc}
\hline \multicolumn{1}{c}{ Parámetros } & TC & NPB & $\begin{array}{c}\text { NPB- } \\
\text { AP100 }\end{array}$ & $\begin{array}{c}\text { NPB- } \\
\text { AP200 }\end{array}$ & SEM $^{1}$ & P $^{2}$ \\
\hline Peso inicial & 52.0 & 58.8 & 59.7 & 62.1 & & \\
Peso final & 84.4 & 90.3 & 91.6 & 94.0 & & \\
Consumo diario, $\mathrm{kg}$ & $1.50^{\mathrm{b}}$ & $2.27^{\mathrm{a}}$ & $1.92^{\mathrm{a}}$ & $2.00^{\mathrm{a}}$ & 0.16 & $<0.01$ \\
Ganancia diaria, $\mathrm{kg}$ & 0.77 & 0.75 & 0.76 & 0.76 & 0.01 & $>0.05$ \\
Conversión Alimenticia & $1.94^{\mathrm{b}}$ & $3.04^{\mathrm{a}}$ & $2.52^{\mathrm{ab}}$ & $2.66^{\mathrm{ab}}$ & 0.23 & $<0.01$ \\
\hline
\end{tabular}

Letras distintas en la misma línea indican diferencia significativa por la Prueba de Tukey

${ }^{1}$ Estándar error of the mean

2 Probabilidad

En relación a la conversión alimenticia el tratamiento testigo presentó el mejor resultado para el análisis consolidado de cerdos en crecimiento y ceba alimentados con bore a voluntad suplementado con núcleo proteico y aceite de palma.

\section{CONCLUSIONES}

La utilización del Tallo de Bore (Alocasia macrorhyza) cocido, en la alimentación porcina es una alternativa para pequeños productores, donde los costos de los tallos no se incrementen por el transporte.

Al suministrar el Bore cocido con el núcleo proteico y sin otra fuente rica en energía, los rendimientos pueden disminuir por el alto consumo del tallo dado su alto contenido de humedad.

El suministro de tallo de bore cocido mejora la ganancia de peso de Cerdos en Levante y Finalización. 
Tabla 5. Rendimiento zootécnico consolidado de cerdos en crecimiento y ceba alimentados con bore a voluntad suplementado con núcleo proteico y aceite vegetal

\begin{tabular}{|c|c|c|c|c|c|c|}
\hline Parámetros & TC & NPB & $\begin{array}{l}\text { NPB- } \\
\text { AP100 }\end{array}$ & $\begin{array}{l}\text { NPB- } \\
\text { AP200 }\end{array}$ & SEM ${ }^{1}$ & $\mathbf{P}^{2}$ \\
\hline Peso inicial & 30 & 31.1 & 31.1 & 33.5 & & \\
\hline Peso final & 84.4 & 90.3 & 91.6 & 94.0 & & \\
\hline Duración, días & 84 & 84 & 84 & 84 & & \\
\hline Consumo diario, $\mathrm{kg}$ & $1.40^{\mathrm{b}}$ & $2.03^{a}$ & $1.70^{a b}$ & $1.70^{a b}$ & 0.14 & $<0.01$ \\
\hline Ganancia diaria, kg & $0.65^{b}$ & $0.71^{a}$ & $0,72^{\underline{a}}$ & $0,72^{a}$ & 0.02 & $<0.03$ \\
\hline Conversión Alimenticia & $2.15^{\mathrm{b}}$ & $2.86^{a}$ & $2.36^{a b}$ & $2.36^{a b}$ & 0.17 & $<0.01$ \\
\hline
\end{tabular}

\section{BIBLIOGRAFÍA}

1. Basto G. Características y efectos del bore en las diferentes fases de la producción porcina. CORPOICA, Bogotá, 32 p. 1995

2. Bermúdez $P$ J E, Rodríguez $P$ J H. Ensilaje de vísceras de cachama blanca (Piaractus brachypomun) como fuente de proteína para la alimentación de cerdos de engorde en una dieta con aceite crudo de palma. Trabajo de Grado Unillanos. 1998.

3. Esquijerosa Y C, García F O, González R H, Pérez N A, Rubalcaba M G P. Comportamiento productivo de cerdos mestizos en ceba alimentados con follaje fresco de Morus alba como sustituto parcial del concentrado comercial. Zootecnia Tropical, 26 (3): 391-394. 2008.

4. González D, González C, Ojeda A, Machado W, Ly J. Comportamiento productivo de cerdos en crecimiento alimentados con jugo de caña (Saccharum officinarum) y harina de follaje de morera (Morus alba). Archivos Latinoamericanos de Producción Animal, 14 (2): 42-43. 2006.

5. Garzón A. V. La producción porcina en el desarrollo agropecuario del piedemonte llanero. Corpoica, Villavicencio, 43 p. 1997: 
6. Garzón A V. Beneficiarios del proyecto con conocimientos y habilidades en el uso de la producción agrícola de la finca Para la alimentación animal. Unidad Instruccional 7. Puerto Carreño, 15 p. 2003.

7. Hauptli L, Lovatto, P A, Silva J H S, Garcia G G, Brum Jr B S e Oliveira, J L S. Níveis de soro de leite integral na dieta de leitões na creche. Ciência Rural, 35 (5): 1161-1165. 2005.

8. Pham S T., Nguyen V. L., Dang H. B. Processing and use of Alocasia macrorrhiza (taro) roots for fattening pigs under mountainous village conditions; Workshop-seminar "Making better use of local feed resources" (Editors: Reg Preston and Brian Ogle) MEKARN-CTU, Cantho, 23-25 May, Article \#44. 2005. Recuperado Febrero 13, 2011, Disponible en: http://www.mekarn.org/proctu/tiep44.htm

9. Pino V E Q, Peña M. Evaluación de tres niveles de harina de bore en dietas para conejos en etapa de crecimiento. Revista Colombiana de Ciencias Pecuarias, 22 (3): 500. 2009.

10. Pinto-Santini L, Escobar A, Messa H F, Ruiz-Silvera C. Evaluación de tres núcleos proteínicos en la dieta de cerdos alimentados con jugo de caña de azúcar y Azolla sp. Livestock Research for Rural Development, 17, Art. \#56. 2005. Recuperado Enero 12, 2011, Disponible en: http://www.Irrd.org//rrd17/5/pint17056.htm

11. Rodríguez L, Lopez D J, Preston T R, Peters K. New Cocoyam (Xanthosoma sagittifolium) leaves as partial replacement for soybean meal in sugar cane juice diets for growing pigs. Livestock Research for Rural Development, 18, Article \#91. 2006. Recuperado Octubre 17, 2010, Disponible en: http://www.Irrd.org//rrd18/7/rodr18091.htm

12. Vélez, J. A. J. El Bore: Nueva alternativa para la Alimentación y la Industria. Agencia Universitaria de Periodismo Científico AUPEC. Cali, 1999. 